\title{
MDM2 controls gene expression independently of p53 in both normal and cancer cells
}

\author{
Giuseppe Arena $^{1} \cdot$ Romain Riscal $^{2,3} \cdot$ Laetitia K. Linares $^{4,5} \cdot$ Laurent Le Cam $^{4,5}$
}

Received: 19 May 2018 / Accepted: 4 June 2018 / Published online: 23 July 2018

(c) ADMC Associazione Differenziamento e Morte Cellulare 2018

The mouse double minute 2 (MDM2) protein is an E3-ubiquitin ligase recognized for its role as a negative regulator of the tumor suppressor protein p53 [1]. Genetic studies showing that embryonic lethality of mice lacking MDM2 could only be rescued by the concomitant deletion of Tp53 suggested that the main physiological role of MDM2 is to inhibit p53. Furthermore, MDM2/p53 double knock-out (KO) mice displayed a cancer incidence similar to that observed in $T p 53 \mathrm{KO}$ animals, supporting the notion that the major role of MDM2 is to interfere with p53 activities [2,3]. However, several reports have challenged this notion and convincingly showed that MDM2 functions extend beyond p53 regulation both in normal and cancer cells.

\section{P53-independent functions of MDM2 in cancer}

A first hint came from the analysis of transgenic mice overexpressing MDM2 in the absence of functional p53. These mice displayed higher incidence of sarcomas compared to p53-null animals, indicating a role for MDM2 in

$\triangle$ Laurent Le Cam

laurent.lecam@inserm.fr

1 Laboratory of Molecular Radiotherapy, INSERM U1030, Gustave Roussy Cancer Campus, Villejuif, France

2 Abramson Family Cancer Research Institute, Perelman School of Medicine, University of Pennsylvania, Philadelphia, PA 19104, USA

3 Department of Cancer Biology, Perelman School of Medicine, University of Pennsylvania, Philadelphia, PA 19104, USA

4 IRCM, Institut de Recherche en Cancérologie de Montpellier, INSERM U1194, Institut régional du Cancer de Montpellier, Université de Montpellier, Montpellier F-34298, France

5 Equipe Labélisée Ligue contre le Cancer, Paris, France tumorigenesis independent of p53 [4]. This notion was further supported by the ability of MDM2 to transform p53-null cells in vitro [5] and by the identification of several MDM2 splice variants that are unable to bind p53 in certain tumor types [6]. Moreover, although rare, some soft tissue tumors exhibit both overexpression of MDM2 and mutations of $T p 53$, a feature that correlates with a higher tumor grade and a poorer prognosis compared to cancer patients harboring only one of these genetic alterations [7].

Different mechanisms through which MDM2 could affect the process of tumorigenesis independently of p53 have been proposed, including: (1) ubiquitination and consequent proteasomal degradation of other proteins involved in tumor suppression, (2) induction of genomic instability and DNA damage, and (3) stabilization of mRNAs encoding pro-tumorigenic/metastatic factors [1, 8]. Several reports have also highlighted a role for MDM2 in transcriptional regulation and epigenetics. It is noteworthy that, depending on the cellular context and upon specific stress conditions, chromatin-bound MDM2 can activate or repress the execution of both p53-dependent and p53-independent transcriptional programs. Already recognized as a repressor of p53-mediated transcription and as a post-translational regulator of histones at p53-responsive genes, MDM2 is also able to regulate gene expression in a p53-independent manner [9]. Thus, on the one hand, findings from Dobbelstein and colleagues indicated that chromatin-bound MDM2 transcriptionally represses the expression of lineage-specific genes through its physical interaction with components of the polycomb repressive complex 2 (PRC2) to promote stemness and cancer cell survival [10]. On the other hand, our group previously demonstrated that upon deprivation of exogenous serine and glycine, MDM2 is recruited to chromatin in a p53-independent manner to induce the expression of genes implicated in de novo serine synthesis, amino-acid metabolism and transport as well as in glutathione homeostasis. In this experimental context, MDM2 recruitment to its target genes requires the transcription factors ATF3/4 


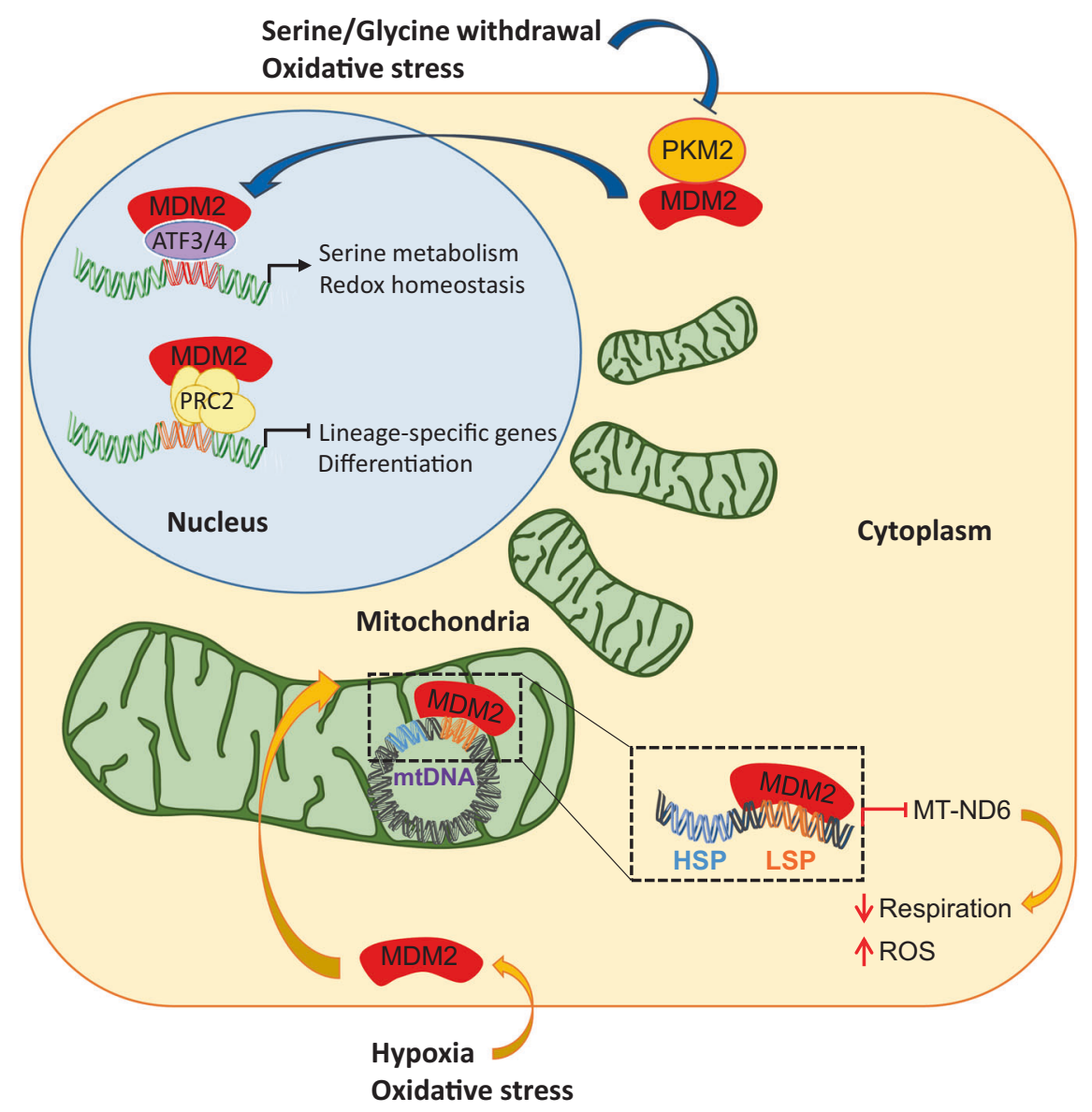

Fig. 1 Schematic representation of p53-independent functions of MDM2 in transcription. Upon specific stress conditions, MDM2 can be recruited to chromatin and/or to the mitochondrial matrix. In basal conditions, MDM2 is sequestered in the cytosol by the pyruvate kinase M2 isoform (PKM2); following serine/glycine deprivation or upon oxidative stress, PKM2 inhibition promotes MDM2 translocation to chromatin at ATF3/4 responsive genes to induce the expression of genes involved in de novo serine synthesis, amino acid transport and glutathione homeostasis. Depending on the cellular context, chromatin-bound MDM2 is also able to inhibit gene expression, as

and is negatively regulated by an important metabolic enzyme, the M2-isoform of pyruvate kinase (PKM2) (Fig. 1). Consistent with this finding, interfering with MDM2 function when cancer cells face a limited availability of exogenous serine and glycine synergistically decreased tumor growth in xenograft assays, suggesting that MDM2-mediated control of serine/glycine metabolism represents an important driver of oncogenesis in tumors overexpressing MDM2 [11].

Underlying the importance of MDM2 in the adaptation of cancer cells to their environmental conditions, we recently found that, under hypoxia, a fraction of cytoplasmic MDM2 also translocates in the mitochondrial matrix to control the transcription of the mitochondrial genome independently of p53. Mitochondrial MDM2 demonstrated by its interaction with the polycomb repressor complex 2 (PRC2) which results in transcriptional repression of lineage-specific genes. Under hypoxic conditions or oxidative stress, a fraction of MDM2 is actively translocated into the mitochondrial matrix. Mitochondrial MDM2 selectively binds to the mtDNA light strand promoter (LSP) and impairs transcription of MT-ND6, a gene encoding a subunit of mitochondrial electron transport chain complex I (CI). By reducing the expression of MT-ND6, mitochondrial MDM2 specifically impinges on CI activity and CI-driven respiration, resulting in accumulation of mitochondrial ROS

(mtMDM2) specifically binds the light strand promoter (LSP) of mitochondrial DNA (mtDNA) and repress the LSP-driven transcription of $M T-N D 6$, a mitochondrial gene encoding an important structural subunit of complex I (CI) of the electron transport chain (ETC) (Fig. 1) [12]. mtMDM2-mediated inhibition of CI-driven respiration causes a significant increase in mitochondrial ROS production, an event previously associated with enhanced invasiveness and metastatic dissemination of cancer cells [13]. Consistent with this notion, lung cancer cells overexpressing a mitochondrial-targeted MDM2 mutant exhibited increased motility and pronounced migratory features. Thus, both chromatin-bound and mitochondrial MDM2 seem to contribute to carcinogenesis but further studies are required to better understand whether these 2 
pools of MDM2 operate in parallel or are differentially activated during distinct phases of cancer progression. Some, but not all, types of oxidative stress can trigger the recruitment of MDM2 both in mitochondria and to chromatin, suggesting a coordinated action of these two pools of MDM2 protein that may contribute to the global metabolic reprogramming of cancer cells. However, it is also possible that the two MDM2 pools respond to distinct changes of the tumor microenvironment and play a complementary role at different steps of cancer progression in order: (1) to sustain cancer cell proliferation during amino acid starvation (chromatin-bound MDM2), and (2) to promote cancer cell migration and metastasis under conditions of low tumor oxygenation (mitochondrial MDM2). Notably, not only p53 but also the E3-ligase activity of MDM2 are dispensable for the transcriptional activities of both chromatin-bound and mtMDM2 on its metabolic and mitochondrial target genes, reinforcing the notion that MDM2 oncogenic activities extend beyond the degradation of $\mathrm{p} 53$.

\section{Physiological roles of MDM2 independent of p53}

Although the importance of serine and glycine metabolism regulation by chromatin-bound MDM2 in normal tissues remains to be determined, our data unambiguously show that accumulation of MDM2 in mitochondria under hypoxic conditions occurs not only in cancer cells but also in nontransformed cells. Thus, the amount of mtMDM2 increased in skeletal muscles of mice exposed to mild hypoxia, and correlated with decreased MT-ND6 mRNA and protein levels. Accordingly, mice lacking both MDM2 and p53 in their hind limbs displayed increased MT-ND6 levels and enhanced mitochondrial complex I activity relative to their corresponding p53 null control animals. As a consequence, MDM2-deficient mice exhibited increased endurance capacity in mild hypoxic conditions, a phenotype that was observed even in the absence of p53 [12]. Whether MDM2 recruitment to mitochondria occurs in other tissues than skeletal muscles and contributes to normal tissue homeostasis is currently under investigation. It is also tempting to speculate that deregulation of mtMDM2 activities plays an important role in pathologies other than cancer. One potential example is in Parkinson disease, a neurological disorder associated with ETC CI defects [14]. Our data also open new avenues to evaluate whether perturbations of mtMDM2 activities are causally involved in mitochondriopathies of unknown etiology. Moreover, the recent striking observation that chronic exposure to hypoxia markedly increased the survival and improved the phenotypic manifestations of a mouse model of Leigh syndrome [15], a pediatric mitochondrial disease commonly associated with ETC CI deficiency, raises interesting questions regarding the potential interest of pharmacological modulation of mtMDM2 activities for new therapies.

\section{Compliance with ethical standards}

Conflict of interest The authors declare that they have no conflict of interest.

\section{References}

1. Biderman L, Manley JL, Prives C. Mdm2 and MdmX as regulators of gene expression. Genes Cancer. 2012;3:264-73.

2. Jones SN, Roe AE, Donehower LA, Bradley A. Rescue of embryonic lethality in Mdm2-deficient mice by absence of p53. Nature. 1995;378:206-8.

3. Montes de Oca Luna R, Wagner DS, Lozano G. Rescue of early embryonic lethality in mdm2-deficient mice by deletion of p53. Nature. 1995;378:203-6.

4. Jones SN, Hancock AR, Vogel H, Donehower LA, Bradley A. Overexpression of Mdm2 in mice reveals a p53-independent role for Mdm2 in tumorigenesis. PNAS. 1998;95:15608-12.

5. Dubs-Poterszman MC, Tocque B, Wasylyk B. MDM2 transformation in the absence of p53 and abrogation of the p107 G1 cellcycle arrest. Oncogene. 1995;11:2445-9.

6. Sigalas I, Calvert AH, Anderson JJ, Neal DE, Lunec J. Alternatively spliced mdm2 transcripts with loss of p53 binding domain sequences: transforming ability and frequent detection in human cancer. Nat Med. 1996;2:912-7.

7. Cordon-Cardo C, Latres E, Drobnjak M, Oliva MR, Pollack D, Woodruff JM, et al. Molecular abnormalities of mdm2 and p53 genes in adult soft tissue sarcomas. Cancer Res. 1994;54:794-9.

8. Bohlman S, ManfredI JJ. p53-independent effects of MDM2. Subcell Biochem. 2014;85:235-46.

9. Wienken M, Moll UM, Dobbelstein M. Mdm2 as a chromatin modifier. J Mol Cell Biol. 2017;9:74-80.

10. Wienken M, Dickmanns A, Nemajerova A, Kramer D, Najafova $\mathrm{Z}$, Weiss $\mathrm{M}$, et al. MDM2 associates with polycomb repressor complex 2 and enhances stemness-promoting chromatin modifications independent of p53. Mol Cell. 2016;61:68-83.

11. Riscal R, Schrepfer E, Arena G, Cissé MY, Bellvert F, Heuillet M, et al. Chromatin-bound MDM2 regulates serine metabolism and redox homeostasis independently of p53. Mol Cell. 2016;62: 890-902.

12. Arena G, Cissé MY, Pyrdziak S, Chatre L, Riscal R, Fuentes M, et al. Mitochondrial MDM2 regulates respiratory complex I activity independently of p53. Mol Cell. 2018;69:594-609.

13. Ishikawa K, Takenaga K, Akimoto M, Koshikawa N, Yamaguchi A, Imanishi $\mathrm{H}$, et al. ROS-generating mitochondrial DNA mutations can regulate tumor cell metastasis. Science. 2008;320:661-4.

14. Schapira AH, Cooper JM, Dexter D, Clark JB, Jenner P, Marsden CD. Mitochondrial complex I deficiency in Parkinson's disease. J Neurochem. 1990;54:823-7.

15. Jain IH, Zazzeron L, Goli R, Alexa K, Schatzman-Bone S, Dhillon $\mathrm{H}$, et al. Hypoxia as a therapy for mitochondrial disease. Science. 2016;352:54-61. 